\title{
Development of an Analytical Method for Kasugamycin Residue in Herbal Medicine, Achyranthes japonica Nakai
}

\author{
Jeong Yoon Choi ${ }^{1}$, Hun Ju Ham², Min-woo Kim ${ }^{1}$, Abd Elaziz Sulieman Ahmed Ishag ${ }^{1,3}$, and Jang-Hyun \\ Hur $^{1^{*}}$ \\ ${ }^{1}$ Department of Biological Environment, Kangwon National University, Chuncheon 24341, Republic of Korea \\ ${ }^{2}$ Environment Friendly Agricultural Products Safety Center, Chuncheon 24341, Republic of Korea \\ ${ }^{3}$ Department of Crop Protection, University of Khartoum, Khartoum North, Shambat 13314, Sudan
}

\section{* Corresponding author:}

email: hurpub303@gmail.com

Received: May 19, 2021

Accepted: October 12, 2021

DOI: $10.22146 /$ ijc. 65970

\begin{abstract}
This study developed a suitable analytical method for kasugamycin residues in Achyranthes japonica using LC/MS/MS equipped with an amide column for polar substances. Extraction and cleanup processes were done at $p H$ 4.5-5. Purification efficiency was assessed and confirmed step by step by selecting silica, hydrophiliclipophilic balance (HLB), strong cation exchange (SCX), and double (HLB and SCX) cleanup SPE cartridges. The results indicated that silica SPE cartridge exhibited overloading tendency, while HLB SPE cartridge had low cleaning efficiency. Among SPE cartridges used, double cleanup and SCX were found sufficient with respective matrix effects of $-15 \%$ and $+14 \%$, respectively. The LOD and LOQ were $0.008 \mathrm{ng}$ and $0.04 \mathrm{mg} / \mathrm{kg}$, respectively. The correlation coefficient $\left(R^{2}\right)$ was higher than 0.99 , recovery rate ranges were $86.3-97.2 \%$, and the RSD was below $8.8 \%$. All methods are consistent with the Codex guidelines criteria. This study developed an appropriate LC/MS/MS analytical method for kasugamycin residue analysis in A. japonica with optimized, efficient extraction and purification conditions using a single SCX SPE cartridge, which is simple and time-efficient. In addition, the HLB and SCX SPE cartridges of the double cleanup methods were identified as primary methods that can be applied for the cleanup of other medicinal herbs.
\end{abstract}

Keywords: LC-MS/MS analysis; Kasugamycin residues; Achyranthes japonica; SPEcartridges

\section{- INTRODUCTION}

Herbal medicine uses medicinal crops' dried roots and leaves for various purposes such as health promotion and disease treatment [1]. The herbal medicine Achyranthes japonica Nakai (Japanese chaff flower) is a perennial herb belonging to the Amaranthaceae family. $A$. japonica contains saponin and a large amount of inflammation-relieving ingredients; thus, it is widely used for arthritis and as a diuretic and tonic [2]. However, in the fall, $A$. japonica is frequently affected by a powdery mildew disease caused by Albugo achyranthis (P. Henn.). This disease could be controlled using a fungicide of copper sulfate basic, copper oxychloride with metalaxyl-m, and copper oxychloride with kasugamycin [3]. Among these, kasugamycin of the aminoglycoside family is a subject of social interest because it can have a potentially adverse effect on the treatment of diseases in humans and livestock due to the occurrence of antibioticresistant bacteria [4].

Kasugamycin, developed as an agricultural fungicide, is a highly polar substance with a $\log \mathrm{P}_{\text {ow }}$ value of -5.75 and is a dissociative compound with carboxy and amino groups in the molecule [5]. The pKa of the carboxylic acid (pKa1), primary cyclic amine (pKa2), and secondary amine (pKa3) are 3.23, 7.73, and 11.0 , respectively, which is characterized by ionization of certain areas in various $\mathrm{pH}$ ranges [6]. The solubility of 
kasugamycin is $228 \mathrm{~g} / \mathrm{L}$ in water $\left(\mathrm{pH} 7,25^{\circ} \mathrm{C}\right)$ and 7.44 $\mathrm{mg} / \mathrm{L}$ in methanol $\left(25^{\circ} \mathrm{C}\right)$. However, it is hardly soluble in most organic solvents. Moreover, kasugamycin is nonvolatile and is physically and chemically unstable in heat; thus, its analysis process is difficult due to its low sensitivity during analysis [5]. According to previous studies, biological potency assays (cup, standard strain Pseudomonas fluorescens) and capillary electrophoresis (CE) were performed, but there were disadvantages in that the analysis time was long, and there were poor precision and selectivity [7]. Also, in the case of HPLC and GLC, which are often used for residual analysis, HPLC/UV showed low absorbance because there was no chromophore for ultraviolet or fluorescence, so there was a sensitivity problem. In addition, it has been reported that the time required for pretreatment is long, and reproducibility is low [5-6,8]. In particular, it has been reported that aminoglycoside-based components are challenging to analyze using GLC without the derivatization of amino and hydroxyl groups due to the hydrophilicity and non-volatile nature of the molecule [9].

On the other hand, studies that analyze kasugamycin on agricultural samples using LC/MS/MS have been conducted steadily recently, with excellent selectivity and sensitivity. Several reports confirmed using various SPE methods to remove interfering substances from samples and improve the sensitivity of kasugamycin. Many authors reported the purification of peppers and soil extracts using an MCX SPE cartridge [7,10]. A study of simultaneous analysis of agricultural fungicides using an HLB SPE cartridge and MCX SPE cartridge was reported. The simultaneous analysis of kasugamycin and validamycin-A through a consecutive cleanup process combined HLB SPE cartridge and SCX SPE cartridge as described in previous studies [8], and simultaneous analysis of kasugamycin and streptomycin in five vegetables was reported [11].

Furthermore, research conducted by the Korean Ministry of Food and Drug Safety (MFDS) to extract kasugamycin with methanol ( $\mathrm{pH}$ 13) for five representative agricultural products, namely brown rice, soybeans, peppers, potatoes, and tangerines, purify it with an HLB SPE cartridge, and conduct analysis using LC/MS/MS was reported [12]. As described above, there are various established methods for analyzing kasugamycin residues in agricultural products in the previous studies; however, there is no report of residual analysis for kasugamycin in herbal medicines containing complex active ingredients. Therefore, this study aims to establish a residual analysis method for the kasugamycin in herbal medicine for the first time in this field by selecting A. japonica Nakai among herbal medicines with various pharmacological components. In particular, to minimize the interference effect that may occur due to the complex matrix in herbal medicine ( $A$. japonica Nakai); by systematically comparing and analyzing the purification efficiency of various SPE cartridges, including extraction and distribution processes. Furthermore, the analysis method also was intended to confirm whether it conforms to the analysis criteria of pesticide residues of the International Food Standards Commission (Codex Alimentarius Commission, CAC/GL 40) and the Guideline on Standard Procedure for Preparation of Test Methods for Food [13] of the Korean MFDS.

\section{- EXPERIMENTAL SECTION}

\section{Reagents and Instruments}

The standard product for the analysis of kasugamycin was bought from Sigma-Aldrich (USA) and had a purity of $98.7 \%$ or higher. The HPLC grade solvents used in the analysis process were water, dichloromethane, acetonitrile, and methanol, purchased from Thermo Fisher Scientific (USA). Formic acid (98.0\%) and ammonia solution were purchased from Sigma-Aldrich (USA) and Daejeonghwageum (Korea), respectively. Also, other organic solvents and reagents were purchased and used for residue analysis. For purification, SPE cartridges, silica ((55 $\mu \mathrm{m}, 70 \AA), 1 \mathrm{~g})$ and SCX ((55 $\mu \mathrm{m}, 70 \AA), 1 \mathrm{~g})$ obtained from Phenomenex (USA), and HLB ((60 $\mu \mathrm{m}, 80 \AA)$, $500 \mathrm{mg})$ purchased from Waters (USA) were used. For uniform extraction, a sonicator (Jeio Tech. Co. Ltd., Korea) was used for sample extraction, and a centrifuge (Allegra X1R, Beckman Coulter Life Sciences, USA) was used for 
centrifugation. In the purification process, a vacuum manifold (Visiprep SPE vacuum manifold, Supelco, USA) and vacuum pump (DOA-P704-AC, Gast, USA) were used for the SPE cartridge. Kasugamycin is highly soluble in water or methanol and ionized in wide $\mathrm{pH}$ ranges. The physicochemical properties and chemical structural formulas of kasugamycin are shown in Table $1[12,14]$.

\section{Procedure}

\section{Preparation of standard solution}

A stock solution was prepared by accurately taking $10.13 \mathrm{mg}$ of the standard compound (98.7\%) of kasugamycin, placing it in a $100 \mathrm{~mL}$ volumetric flask, and then dissolving it in water to make $100 \mu \mathrm{g} \mathrm{mL}^{-1}$. In the same way, the working solution was diluted stepwise to concentrations of $0.005,0.008,0.01,0.05,0.08$, and $0.1 \mu \mathrm{g} \mathrm{mL}$. For the standard calibration curve for quantification, the matrix was included in a 1:1 ratio of the standard solution and the extraction solution to create a matrix-matched calibration curve. Since the target component is an aminoglycoside-based fungicide that has the property of adsorbing to glass, all containers used in the analysis were made of polypropylene [12].

\section{Herbal medicine sample}

The herbal medicine, Achyranthes japonica Nakai, was purchased from a pharmaceutical company (Iksan, Jeollabuk-do) that applied distribution according to the drug specifications and was used after verification and a sensory evaluation a herbal medicine expert. Since the roots are used as medicinal herbs, the dried roots were ground and passed through a $2 \mathrm{~mm}$ sieve, and then a $5 \mathrm{~g}$ sample was weighed and used for analysis.

\section{Extraction and distribution}

Ten $\mathrm{mL}$ of distilled water was added to $5 \mathrm{~g}$ of the dried roots sample, and the wetting process was performed for $1 \mathrm{~h}$. The extraction solvent was used after adjusting the $\mathrm{pH}$ to $4.5-5$ by adding $0.1 \%$ formic acid to methanol, methanol/water (5/5, v/v), and water. Sample extraction was performed by ultrasonic extraction using a sonicator for $30 \mathrm{~min}$, followed by centrifugation for $10 \mathrm{~min}$ at $4{ }^{\circ} \mathrm{C}$ and $4,000 \mathrm{G}$. The liquid-liquid distribution process was carried out by taking $5 \mathrm{~mL}$ of the supernatant when using water in the extraction solvent and then adding $5 \mathrm{~mL}$ of dichloromethane.

\section{Purification conditions using SPE cartridge}

In this study, a comparative experiment was conducted using various SPE cartridges to establish the optimal purification conditions for kasugamycin in $A$. japonica. The SPE cartridges used during the experiment were silica, HLB, and SCX SPE cartridges. The purification conditions are as follows: In the SPE cartridge refining process, a vacuum manifold device equipped with a vacuum pump was used to allow elution at a flow rate of one to two drops per second to maintain a constant flow rate and shorten the time required.

Silica SPE cartridge purification conditions. After activating the filler, $3 \mathrm{~mL}$ of methanol and water was

Table 1. Physicochemical properties of kasugamycin

\begin{tabular}{ll}
\hline IUPAC name & $\begin{array}{l}\text { 2-amino-2-[(2R,3S,5S,6R })-5 \text {-amino-2-methyl-6-[(2R,3S,5S,6S)-2,3,4,5,6- } \\
\text { pentahydroxycyclohexyl]oxyoxan-3-yl]iminoacetic acid }\end{array}$ \\
\hline Chemical structure & 379.4 \\
\hline $\mathrm{V} \cdot \mathrm{p} .(\mathrm{mPa})$ & $<1.3 \times 10^{-4} \mathrm{~mm} \mathrm{Hg}\left(25^{\circ} \mathrm{C}\right)$ \\
\hline $\mathrm{K}_{\mathrm{ow}} \operatorname{logP}$ & -5.75 \\
\hline
\end{tabular}


added to the silica SPE cartridge (1 g), $1 \mathrm{~mL}$ of the extraction supernatant was adsorbed onto the cartridge. After that, $10 \mathrm{~mL}$ of water was sequentially discharged, and kasugamycin was eluted using $10 \mathrm{~mL}$ of methanol.

\section{HLB SPE cartridge purification conditions.} Hydrophilic lipophilic balance (HLB) was used after activating the filler with $3 \mathrm{~mL}$ of methanol and water in the SPE cartridge $(500 \mathrm{mg})$. Then, $5 \mathrm{~mL}$ of the extraction supernatant was loaded into the cartridge; $5 \mathrm{~mL}$ of methanol was added and combined with the previously fractionated eluate to obtain a sample.

SCX SPE cartridge purification conditions. After activating the filler using $5 \mathrm{~mL}$ of water and methanol in the SCX SPE cartridge $(1 \mathrm{~g}), 2 \mathrm{~mL}$ of the extracted supernatant was adsorbed to the cartridge. After that, $10 \mathrm{~mL}$ of water and $10 \mathrm{~mL}$ of methanol were sequentially discharged, and kasugamycin was eluted using $10 \mathrm{~mL}$ of methanol, to which $5 \% \mathrm{NH}_{4} \mathrm{OH}$ was added.

\section{LC/MS/MS instrument analysis}

The LC-20AXR series (Shimadzu, Japan) was used as an analytical instrument for HPLC, and the TSQ Quantum Ultra (Thermo Scientific, USA) was used for mass spectrometry. The columns used included an Imtakt C18 column ( $2 \mathrm{~mm}$ ID $\times 100 \mathrm{~mm}, 3.0 \mu \mathrm{m}$, USA), Waters Hydrophilic Interaction Liquid Chromatography (HILIC) column ( $3.0 \mathrm{~mm}$ i.d. $\times 100 \mathrm{~mm}, 3.5 \mu \mathrm{m}$, USA), and Xbridge amide column $(3.0 \mathrm{~mm}$ i.d. $\times 100 \mathrm{~mm}, 3.5$ $\mu \mathrm{m}$, USA). The oven temperature was fixed at $35^{\circ} \mathrm{C}$. The mobile phase solvents were $0.1 \%$ formic acid in water (A) and $0.1 \%$ formic acid in acetonitrile (B). The flow rate was
$0.4 \mathrm{~mL} / \mathrm{min}$, and the injection volume was $5.0 \mu \mathrm{L}$. The gradient conditions were set as the following: (1) $20 \% \mathrm{~A}$ and $80 \%$ B held for $1.0 \mathrm{~min}$, (2) 60\% A and $40 \% \mathrm{~B}$ for 4.0 min, (3) $90 \% \mathrm{~A}$ and $10 \% \mathrm{~B}$ for $2.0 \mathrm{~min}$, (4) $20 \% \mathrm{~A}$ and $80 \%$ B for $0.5 \mathrm{~min}$, and finally, (5) $20 \% \mathrm{~A}$ and $80 \%$ B for $2.5 \mathrm{~min}$. The overall running time was $10.0 \mathrm{~min}$. The MS/MS was analyzed by the multiple reactions monitoring (MRM) method using electrospray ionization (ESI) positive mode, a spray voltage of $4.0 \mathrm{kV}$, and capillary and vaporizer temperatures of 280 and 300 ${ }^{\circ} \mathrm{C}$, respectively. The Ion sweep gas pressure was set to 1.5 Arb. Sheath gas pressure was 40 units, and Aux gas pressure was 30.0 units. The precursor and product ions were monitored (Table 2).

\section{Test method validation}

The established test method for kasugamycin was verified using the detection and quantitation limits of the device, the quantitative limit of the test method, linearity, accuracy, and repeatability. As for the instrument's detection limit, a concentration in which the signal-to-noise ratio (signal/noise, $\mathrm{S} / \mathrm{N}$ ) of the peak detected on the chromatogram was 3 or more, and the quantitative limit was 10 or more. The limit of quantification of the test method was calculated by taking into account the minimum detection amount, sample amount, test solution amount, and dilution ratio in instrument analysis (Eq. (1)). Linearity was evaluated by making a blank line using a matrix-matched standard solution and calculating the correlation of determination $\left(\mathrm{R}^{2}\right)$. The suitability, precision, and repeatability of the

Table 2. LC/MS/MS operating parameters for the analysis of kasugamycin

\begin{tabular}{|c|c|c|c|}
\hline HPLC & \multicolumn{3}{|c|}{ LC-20AXR series (Shimadzu, Japan) } \\
\hline Detector & \multicolumn{3}{|c|}{ TSQ Quantum Ultra (Thermo Science, USA) } \\
\hline & \multicolumn{3}{|c|}{ (1) Imtakt $C_{18}$ column ( 2 mm I.D. $\left.\times 100 \mathrm{~mm}, 3.0 \mu \mathrm{m}, \mathrm{USA}\right)$} \\
\hline Column & \multicolumn{3}{|c|}{$\begin{array}{l}\text { (2) Waters HILIC column ( } 3.0 \mathrm{~mm} \text { I.D. } \times 100 \mathrm{~mm}, 3.5 \mu \mathrm{m} \text {, USA) } \\
\text { (3) Xbridge amide column ( } 3.0 \mathrm{~mm} \text { I.D. } \times 100 \mathrm{~mm}, 3.5 \mu \mathrm{m}, \mathrm{USA})\end{array}$} \\
\hline Oven temp. & \multicolumn{3}{|l|}{$35^{\circ} \mathrm{C}$} \\
\hline \multirow{4}{*}{ Scan events } & Precursor ion & Product ions & $\mathrm{CE}$ \\
\hline & \multirow{3}{*}{380.093} & 111.993 & 32 \\
\hline & & 156.002 & 33 \\
\hline & & 199.963 & 33 \\
\hline
\end{tabular}


above method were analyzed after adding a standard solution equivalent to the limit of quantification $(0.04$ $\mathrm{mg} / \mathrm{L})$ and the limit of quantitation 10 times $(0.4 \mathrm{mg} / \mathrm{L})$ to the untreated sample. The recovery rate and analysis error of three repetitions were verified.

$\mathrm{A}(\mathrm{ng}) \times \frac{\mathrm{B}(\mathrm{mL})}{\mathrm{C}(\mathrm{g})} \times \frac{\mathrm{D}(\mathrm{mL})}{\mathrm{E}(\mathrm{mL})} \times \frac{\mathrm{F}}{\mathrm{G}(\mu \mathrm{L})}=\operatorname{MLQQ}(\mathrm{mg} / \mathrm{kg})$

whereas $\mathrm{A}$ is the minimum amount of detection, $\mathrm{B}$ is the volume of extraction solvent, $\mathrm{C}$ is sample amount, $\mathrm{D}$ is the final volume, $\mathrm{E}$ is the supernatant amount taken from the extracted solvent, $F$ is dilution factor, and $\mathrm{G}$ is injection quantity.

\section{Matrix effect (ME) of the purification method}

Matrix effect refers to suppressing or enhancing the component's ionization by affecting the analyte component's instrumental sensitivity due to the target sample [15]. Matrix effect calculation is determined by comparing the calibration curve of the standard solution with the calibration curve of the matrix-matched standard solution, and the formula is shown in Eq. (2). This study calculated the matrix effect after preparing a matrixmatched standard solution of $0.005,0.008,0.01,0.05,0.08$, and $0.1 \mathrm{mg} / \mathrm{kg}$ using untreated samples extracted through each SPE purification process.

Matric effect $(\%)=$

$$
\left(\frac{\text { Slope of calibration curve matrix }}{\text { Slope of calibration curve solvent }}-1\right) \times 100
$$

\section{- RESULTS AND DISCUSSION}

\section{Establishment of LC/MS/MS Analysis Conditions}

In this study, analysis conditions using LC/MS/MS with high sensitivity and high selectivity were established to analyze kasugamycin. For the LC/MS/MS instrument analysis, electrospray ionization (ESI) positive mode was selected, and precursor ion and collision energy were optimized using multiple reaction monitoring (MRM) mode. The parent compound was detected as $[\mathrm{M}+\mathrm{H}]^{+}$in positive mode, and the precursor ion was selected as $\mathrm{m} / \mathrm{z}$ 380.072. The product ion was selected as $\mathrm{m} / \mathrm{z} 111.994, \mathrm{~m} / \mathrm{z}$ 156.003, and $\mathrm{m} / \mathrm{z}$ 199.964. The high intensity $(\mathrm{m} / \mathrm{z}$ 156.003 and $\mathrm{m} / \mathrm{z}$ 199.964) was selected for quantitation (Fig. S1).
Kasugamycin is a polar compound with a $\log \mathrm{P}_{\text {ow }}$ value of -5.75 and high solubility in water or methanol. Besides that, this compound is ionized in a wide range of $\mathrm{pH}$ values [16-17]. Therefore, in this study, water and acetonitrile, commonly used to analyze aminoglycoside antibiotics, were selected as the mobile phase solvent, and $0.1 \%$ formic acid solution was added to improve the sensitivity and resolution efficiency of the target component $[5,18]$. Previous studies found that the sensitivity of the analyte is increased when a $0.1 \%$ formic acid solution is added to the mobile phase, while the sensitivity of the signal decreases when a concentration lower than $0.1 \%$ is added $[8,19]$.

As a result of comparing and analyzing the resolution and sensitivity of kasugamycin through $\mathrm{C} 18$, HILIC, and amide columns for HPLC analysis, the C18 column was rapidly eluted without staying in the column due to the amino group of kasugamycin, and it was not easy to separate it from the interfering material, as shown in Fig. 1(a) $[11,19]$. The HILIC column acts effectively on polar basic compounds and aminoglycosides, but its retention time was short, and peak tailing was confirmed, which required improvement, as seen in Fig. 1(b) [20-21]. An amide column is a form in which polar amide groups are bound [7]. Nevertheless, it was reported that the retention time and sensitivity were excellent, and because of confirming this, sufficient retention time and sensitivity were secured, as shown in Fig. 1(c) $[7,10,19]$. Therefore, in this study, an amide column highly effective for kasugamycin was selected and analyzed.

\section{Establishment of Extraction and Distribution Process}

Dried samples showed low extraction efficiency in aqueous organic solvents, so the wetting process was essential. Thus, $10 \mathrm{~mL}$ of distilled water was added to the dried sample during analysis [22]. The extraction solvent was reported to increase the recovery rate when a small amount of acid was added during the extraction of kasugamycin; accordingly, the $\mathrm{pH}$ of the extraction solvent was maintained between about 4.5-5.5 using a formic acid solution [8]. Therefore, the recovery rates of 


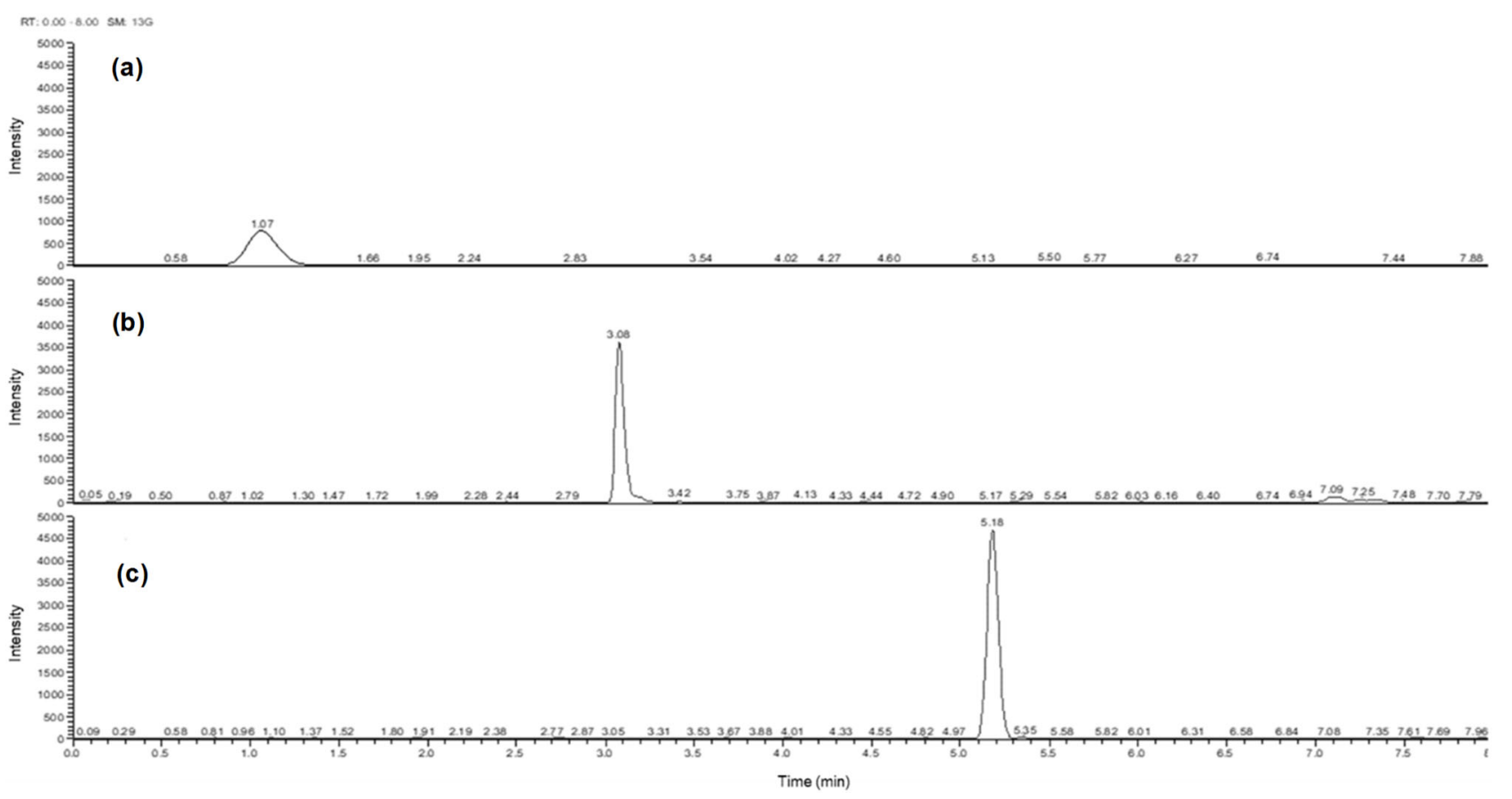

Fig 1. Chromatogram of $0.1 \mathrm{mg} \mathrm{kg}{ }^{-1}$ kasugamycin standard solution compared by columns ((a): $0.1 \mathrm{mg} \mathrm{kg}^{-1}$ of

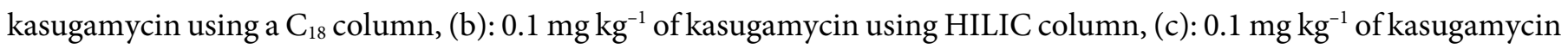
using amide column)

methanol, methanol/water (5:5, v/v), and water were compared to select the optimal extraction solvent when the $\mathrm{pH}$ was maintained at $4.5-5$. The recovery rates were $58.4 \%, 90.2 \%$, and $96.3 \%$, respectively. Water was selected as the final extraction solvent in\% with the highest recovery rate (Table S1). The extraction of kasugamycin from dried samples using methanol and water was previously reported, and the recovery rate found increased as the water content increased [7]. Therefore, in this study, $10 \mathrm{~mL}$ of distilled water was added to the sample $(5 \mathrm{~g})$, followed by a wetting process for $1 \mathrm{~h}$, and then extracted with $40 \mathrm{~mL}$ of water adjusted to $\mathrm{pH} 4.5-5$ by adding $0.1 \%$ formic acid solution. After $30 \mathrm{~min}$ of ultrasonic extraction using a sonicator, centrifugation was performed at $4{ }^{\circ} \mathrm{C}$ and $4,000 \mathrm{G}$ for $10 \mathrm{~min}$. Even after the extraction process, a liquid-liquid distribution process using dichloromethane, an organic solvent with high specific gravity, was added to remove the non-polar interfering substances contained in the sample extracts. It was visually confirmed that suspended matter or precipitates in the actual extract were removed. In addition, since dichloromethane effectively removes a large amount of non-polar substances and substances of fat components, the above analysis method was determined to be effective in removing non-polar substances and interference substances fat components [7]. However, the results in Fig. 2 show that the effect of removing interference substances on the chromatogram was insufficient, so various types of SPE purification methods were attempted for further purification.

\section{Solid-Phase Extraction (SPE) Cartridge Purification}

Interfering substances continued to appear on the chromatogram even after the extraction and distribution process, and based on previous studies, a suitable SPE cartridge of kasugamycin was selected and compared. In this study, a silica SPE cartridge representing the adsorption principle as a normal cartridge was selected and analyzed for the smooth separation and analysis of the large amount of pharmacologically active substances contained in A. japonica and kasugamycin, and the HLB SPE cartridge analyzed for agricultural products by the 


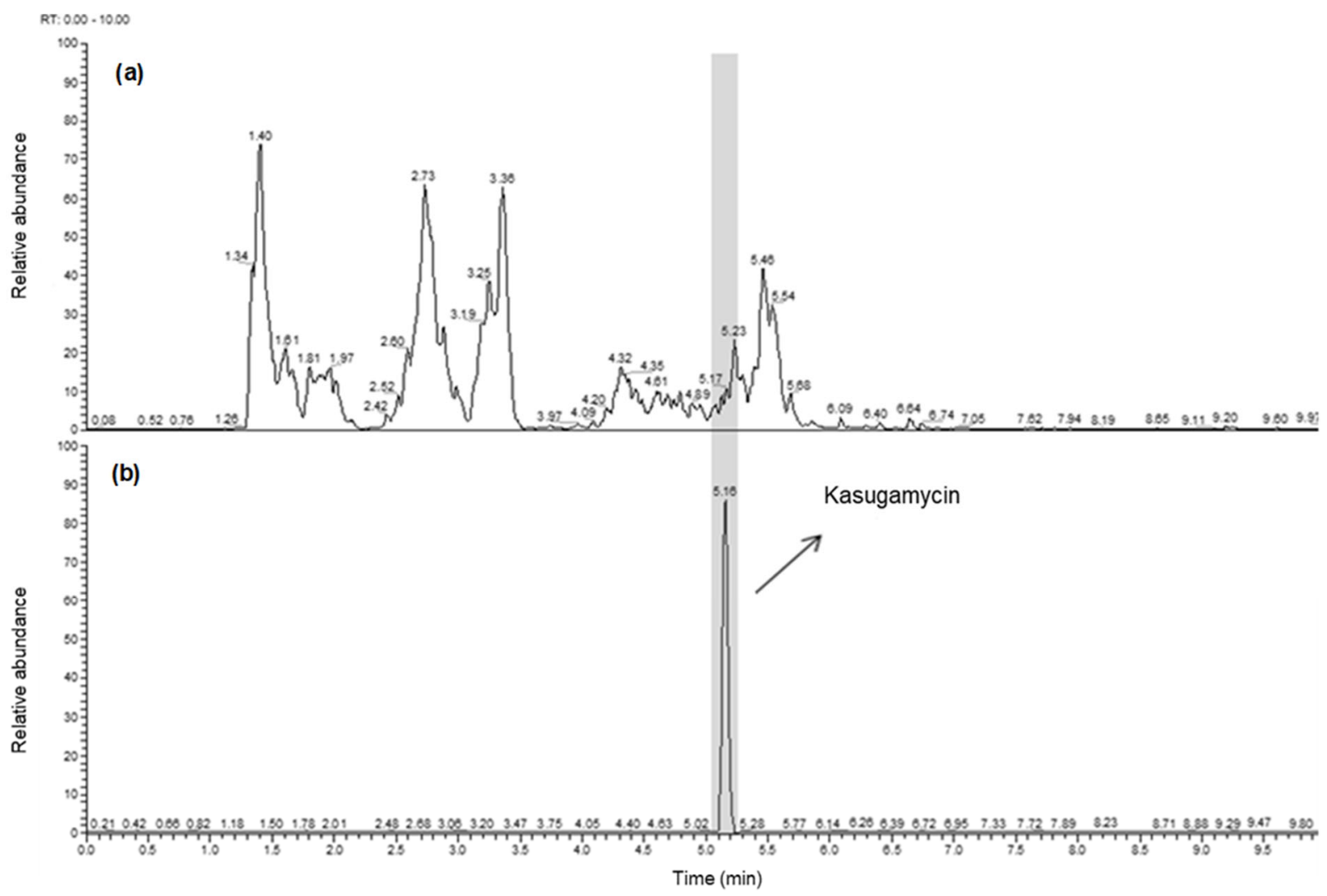

Fig 2. Chromatogram of liquid-liquid partitioning of kasugamycin in Achyranthes japonica Nakai and kasugamycin standard ((a): control, (b): kasugamycin $0.1 \mathrm{mg} \mathrm{kg}^{-1}$ )

MFDS. As a result, it was confirmed that it could be applied to the herbal medicine A. japonica [12]. In addition, when analyzing kasugamycin, the SCX and SCE SPE cartridges were selected and analyzed as recommended by Zhang et al. [8].

\section{Silica SPE cartridge}

The current study is intended to check whether the silica SPE cartridge normally used in the cleanup process during the analysis of pesticide residues can be used to clean kasugamycin, a bio-pesticide used in herbal medicine. The main problem in kasugamycin residue analysis in herbal medicine is the matrix's interference effect, continuously observed after extraction. Therefore, complete removal of A. Japonica roots matrix effect is important and required by the national MRLs [8]. The removal of the matrix effect during kasugamycin residue analysis in herbal medicine is determined using the elution conditions. Therefore, the current study focused on optimizing the elution conditions of kasugamycin residue analysis. The effect of the loading volume of the extract and the volume and type of the eluting solvent indicated that using $1 \mathrm{~mL}$ fortified elution volume with $10 \mathrm{~mL}$ methanol as an eluting solvent on silica SPE cartridge could result in a $93.2 \%$ dissolution rate of kasugamycin (Fig. S2). However, the dissolution rate of kasugamycin was increased to $95.5 \%$ when using $10 \mathrm{~mL}$ of pure water. The lost amount of kasugamycin residue in both cases is attributed to the interfering substances of the matrix causing the overloading of the cartridge and consequently the loss of the target compound (kasugamycin). Several trials were done to minimize the influence of the matrix of the A. Japonica roots of the purification method using a silica SPE cartridge without success. Therefore, another set of trials were done to replace the silica SPE cartridge with HLB SPE and SCX SPE cartridges which were reported to have better efficiency [5,23]. 


\section{HLB SPE cartridge}

The HLB SPE cartridge is known to have high purification efficiency in analyzing aminoglycoside antibiotics. Previous studies reported the analysis of kasugamycin among agricultural products by using an HLB SPE cartridge [12,5]. When using the kasugamycin standard solution, only the HLB SPE cartridge purification gave a recovery rate of $95.8 \%$. When applying $5 \mathrm{~mL}$ of the sample extract to the standard condition, a 97.3\% recovery rate of kasugamycin was obtained (Fig. S3). However, quantification of the minimum concentration was not possible due to the interference substances in the A. japonica roots extract co-eluted with kasugamycin on the chromatogram (Fig. 3). The current result is similar to the results reported by previous authors that the HLB SPE cartridge has low purification efficiency and high ion suppression [7-8]. Therefore, another purification condition was tested using the SCX SPE cartridge, a strong cationic SPE cartridge that exhibits an efficient purification effect for polar substances with many amino and hydroxy groups [8].

\section{SCX SPE cartridge}

The SCX SPE cartridge ( $1 \mathrm{~g})$, a strong ion exchange cartridge, has been reported to effectively purify basic amine compounds from materials with complex matrices [24-25]. In particular, kasugamyacin has various $\mathrm{pKa}$ values, so the purification efficiency is higher when an ion exchange cartridge is used, rather than the florisil or silica SPE cartridge generally used [5]. In addition, the optimal purification method with SCX SPE cartridges has been widely reported. It can sufficiently separate the matrix interfering substance during kasugamycin and validamycin A analysis [18]. The purification conditions with the SCX SPE cartridge were tested using the standard solution, and the target component was not eluted in the loading and washing section. Kasugamycin showed a $98.1 \%$ dissolution rate when $10 \mathrm{~mL}$ of $5 \% \mathrm{NH}_{4} \mathrm{OH}$ in methanol was used. However, when the sample was applied, the recovery rate was $58.8 \%$, which indicates a section lost before the elution section. Therefore, a recovery rate test was performed for each section to solve this problem by applying an A. japonica sample. As a result, about $40.5 \%$ was eluted in the loading and washing sections (Fig. S4).

The overloading phenomena may explain these results due to the matrix effect in the test cartridge as

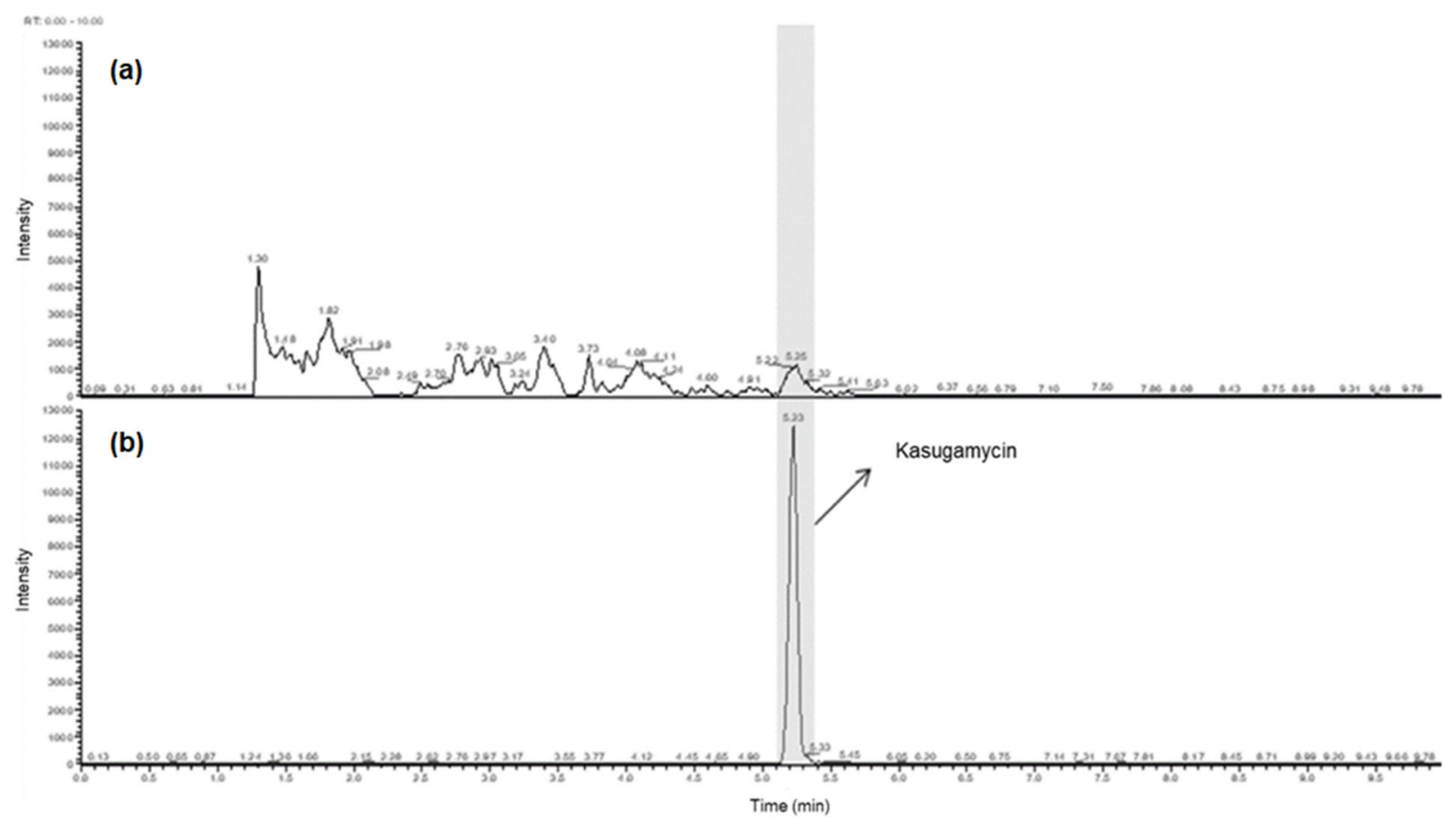

Fig 3. Chromatogram of HLB SPE cartridge test of kasugamycin in Achyranthes japonica Nakai and kasugamycin standard ((a): control, (b): kasugamycin $0.1 \mathrm{mg} \mathrm{kg}^{-1}$ ) 
reported for the silica SPE cartridge [26]. Therefore, a test was conducted using a loading range of 1-4 mL sample extract. The results of the loading capacity experiment showed that the target component was not eluted in the loading and washing sections when $1-3 \mathrm{~mL}$ was used, and the entire amount was eluted in $10 \mathrm{~mL}$ of $5 \% \mathrm{NH}_{4} \mathrm{OH}$ in methanol from the elution section (Table S2). Based on this result, $2 \mathrm{~mL}$ was selected as the optimal loading capacity, and the elution solvent was established under purification conditions using $10 \mathrm{~mL}$ of $5 \% \mathrm{NH}_{4} \mathrm{OH}$ in methanol. The above purification conditions gave a recovery rate of $101.2 \%$, and the interfering substances continuously appearing near the retention time of kasugamycin were removed, and quantification was possible even at the minimum concentration (Fig. 4). Further experiments were done using a consecutive cleanup process combining the HLB SPE cartridge and the SCX SPE cartridge, which was reported to have more efficiency [8].

\section{Double cleanup using HLB SPE cartridge + SCX SPE cartridge}

Based on previous studies, the double cleanup method using the HLB and SCX SPE cartridge was performed. The result indicated a recovery rate in the elution section of $93.7 \%$ using the standard solution (Fig. S3). However, the recovery rate was reduced to $48.5 \%$. Therefore, a second purification was performed using $5 \mathrm{~mL}$ of the sample extract. Overloading is reported to be caused by the difference in sample matrix type used and the cartridge capacity. Therefore, the overloading phenomenon observed in this study may be due to the overwhelming capacity limit of the SCX SPE cartridge by the A. japonica roots extract [27].

Unlike agricultural products, herbal extracts contain various and considerable pharmacological ingredients that saturate the cartridge's adsorption sites. As a result, the normal adsorption and desorption of the analyte components are impossible. Therefore, to overcome overloading from the sample, an additional cleanup process was performed by applying the improved SCX SPE cartridge purification method in which the volume of the extract was reduced from 5 to 2 $\mathrm{mL}$. The result of the improved purification conditions gave a recovery rate in the elution section of $88.4 \%$, and
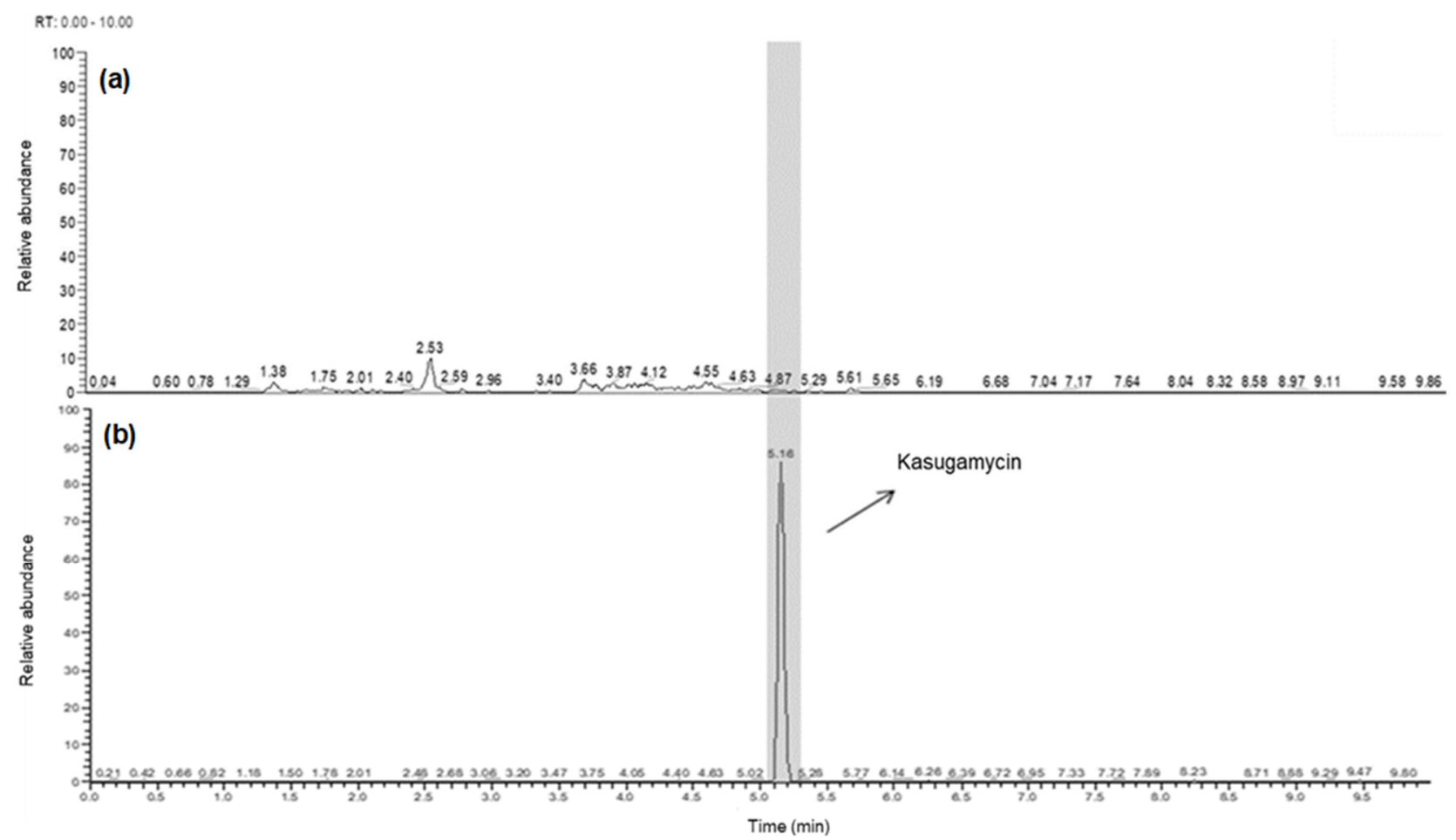

Fig 4. Chromatogram of SCX SPE cartridge test of kasugamycin in Achyranthes japonica Nakai and kasugamycin standard ((a): control, (b): kasugamycin $0.1 \mathrm{mg} \mathrm{kg}^{-1}$ ) 
after double clean-up, the interfering substances eluted around the retention time of kasugamycin disappeared, and therefore that the minimum concentration could be quantified (Fig. 5). Thus, the current results indicated that the SCX SPE cartridge with the double clean-up method, and reduced loading to $2 \mathrm{~mL}$, effectively prevents overloading and removes the herbal extract's interference effects. However, the reduction in loading volume could reduce the representativeness of the sample, therefore not recommended.

\section{Comparison of Matrix Effect (ME) of the Purification Methods}

The matrix effect of each purification method developed for kasugamycin analysis in A. japonica gave different results depending on the cartridge. The matrix effect of the HLB SPE cartridge was $-36 \%$, while those of the SCX SPE cartridge alone and the double clean up with HLB and SCX SPE cartridges were $-15 \%$ and $+14 \%$, respectively (Table 3 ). The two analytical methods of the SCX SPE cartridge were matrix-matched in 1:1 ratio of the standard substance and the extract solution with the lowest possible effect of the interfering substance in the
LC/MS/MS analysis at the lowest possible quantitative level. Since there were no differences in the matrix effect value from the presence or absence of the HLB SPE cartridge, it was assumed that the purification through a non-retentive filter was insufficient, and therefore most of the purification was performed in the SCX SPE cartridge. The consecutive cleanup process that directly connects HLB and SCX SPE cartridges gave excellent superior results. Nevertheless, since purification with a single SCX SPE cartridge gave sufficient purification efficiency and saved time, effort, and the method's simplicity, the current investigation recommends using a single SCX SPE cartridge for the purification of kasugamycin in herbal medicine [8].

\section{Overview of the Method Established for Kasugamycin in Herbal Medicine}

The sample should be wetted for one hour by adding $10 \mathrm{~mL}$ of water to the dry sample and adjusting the $\mathrm{pH}$ to 4.5 to 5.0 using $40 \mathrm{~mL}$ of $0.1 \%$ formic acid in the water extraction solvent. Then, ultrasonic extraction should be performed for $30 \mathrm{~min}$. Then the extract should be centrifuged for $10 \mathrm{~min}, 5 \mathrm{~mL}$ of the supernatant should

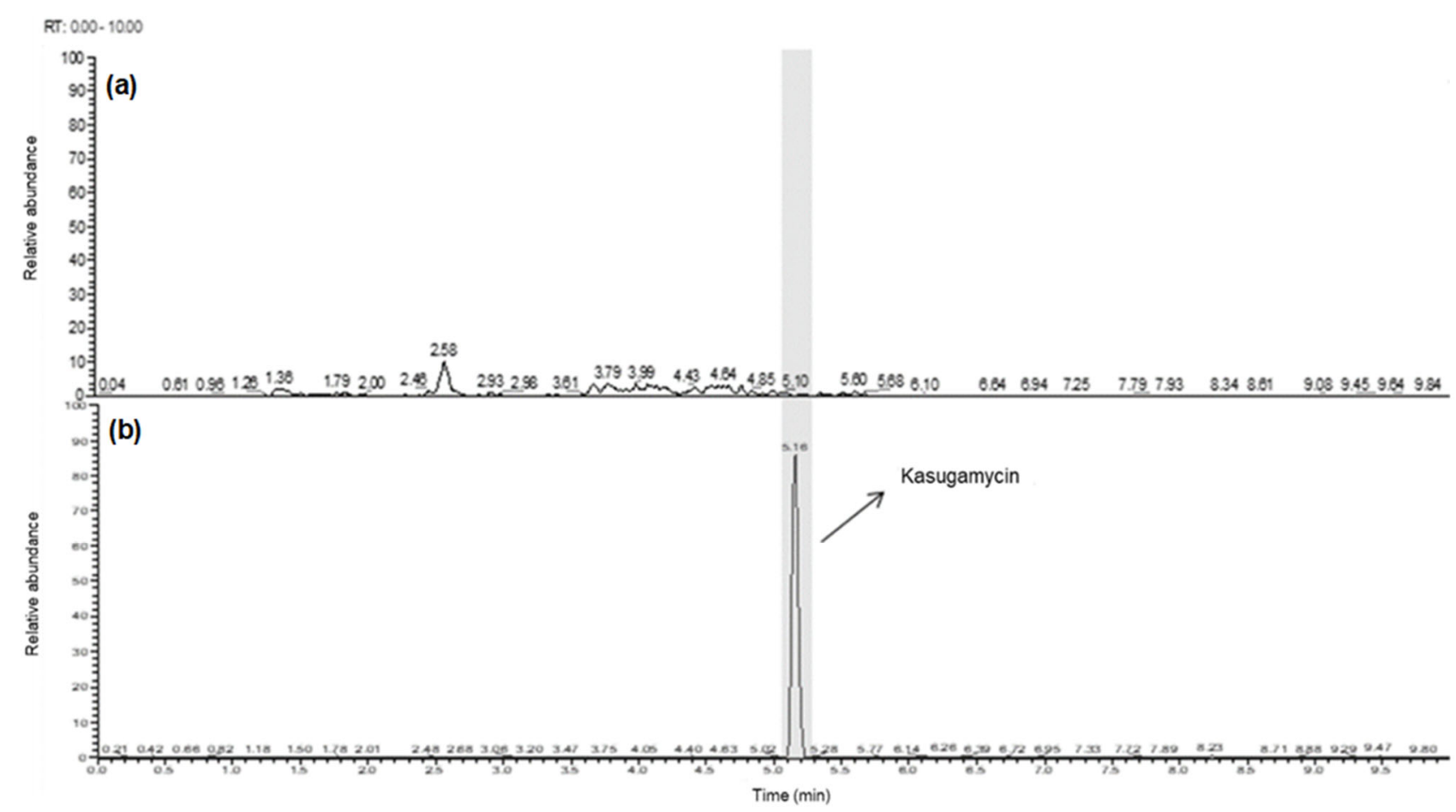

Fig 5. Chromatogram of HLB \& SCX SPE cartridge test of kasugamycin in Achyranthes japonica Nakai and kasugamycin standard ((a): control, (b): kasugamycin $\left.0.1 \mathrm{mg} \mathrm{kg}^{-1}\right)$ 
Table 3. Results of matrix effect (\%) of kasugamycin in Achyranthes japonica Nakai

\begin{tabular}{llll}
\hline Sorbent & Regression equation & Linearity $\left(\mathrm{r}^{2}\right)$ & Matrix effect $(\%)$ \\
\hline Solvent & $\mathrm{y}=265136.035 \mathrm{x}-473.3349723$ & 0.9964 & - \\
HLB & $\mathrm{y}=168547.8359 \mathrm{x}+6578.965386$ & 0.9951 & -36 \\
SCX & $\mathrm{y}=225862.6871 \mathrm{x}-36.41891167$ & 0.9968 & -15 \\
HLB + SCX & $\mathrm{y}=301291.8221 \mathrm{x}+422.1827966$ & 0.9923 & +14 \\
\hline
\end{tabular}

be taken and centrifuged after liquid-liquid partitioning at the same ratio as dichloromethane, then $2 \mathrm{~mL}$ of the supernatant should be used as a loading sample for purification. Purification conditions should be done on the SCX SPE cartridge ( $1 \mathrm{~g})$, a strong ion exchange cartridge. First, the filter should be activated by adding 5 $\mathrm{mL}$ of water and methanol, then loaded with $2 \mathrm{~mL}$ of the sample extract, followed by washing with $10 \mathrm{~mL}$ of water and methanol, and eluting with $10 \mathrm{~mL}$ of $5 \% \mathrm{NH}_{4} \mathrm{OH}$ in methanol. Next, the elution solution should be concentrated completely under a gentle stream of nitrogen, then reconstituted in $1 \mathrm{~mL}$ of water, filtered with a $0.2 \mu \mathrm{m}$ membrane filter, and subject to LC/MS/MS analysis (Fig. 6).

\section{Test Method Validation}

The established method eliminated the effect of the interfering substance co-eluting with kasugamycin and therefore allowed accurate estimation of levels close to the quantification level. As a result of evaluating the selectivity of the test method, when the established analysis method was applied, the effect of interference near the kasugamycin retention time was minimized, and the quantitative limit level was quantifiable. Therefore, it could be said that it was a preferable analysis method. The reliability of the results of residue analysis in A. japonica was by a matrix-matched calibration curve done by diluting the standard solution

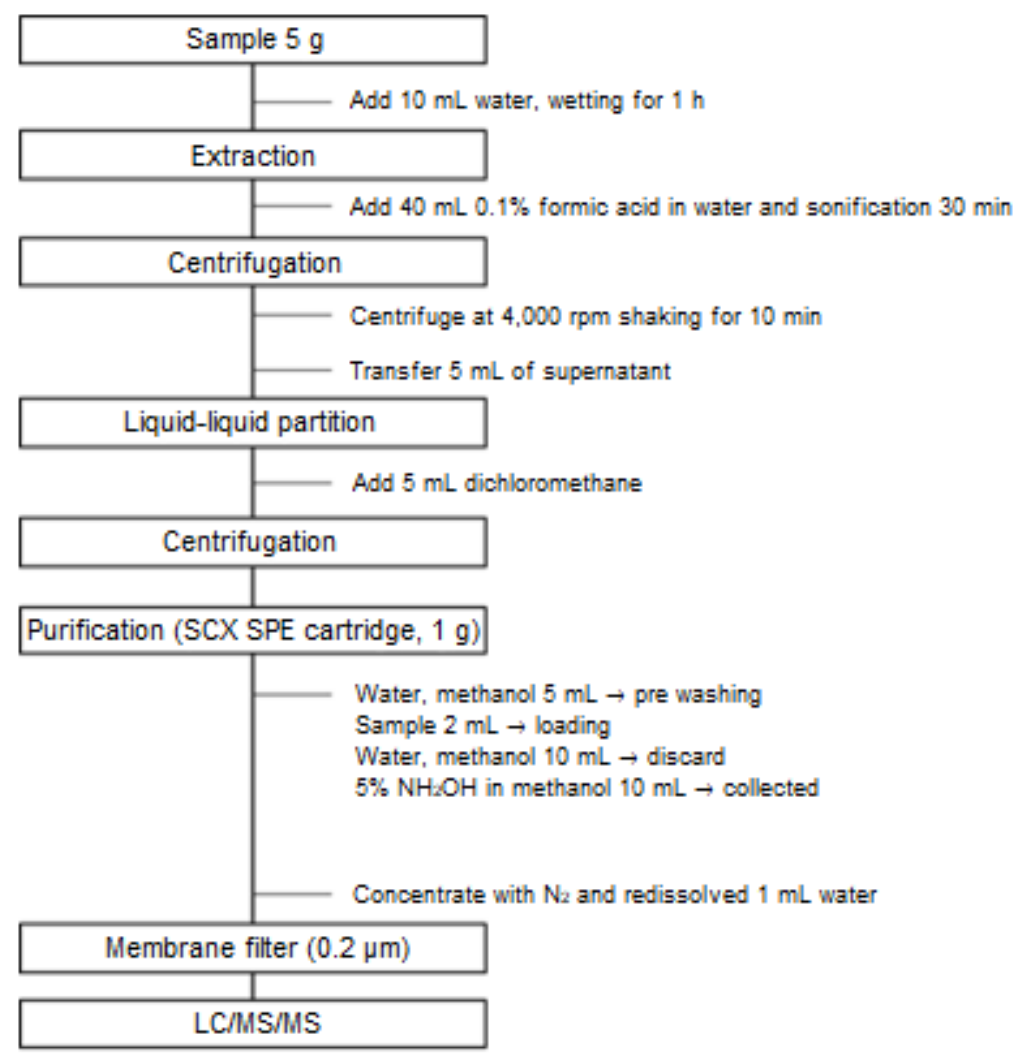

Fig 6. Improved analytical method of kasugamycin residue in Achyranthes japonica Nakai 
of kasugamycin to $0.02,0.05,0.1,0.2,0.5,1.0$, and $2.0 \mathrm{mg} / \mathrm{L}$. The results gave a matrix-matched calibration curve of 0.996 or higher (Fig. 7 and Table S3).

The detection limit (LOD) of the device used in the current study was $0.008 \mathrm{ng}$, while the quantification limit (LOQ) of the test method was $0.04 \mathrm{mg} / \mathrm{kg}$ (Eq. (3)). The LOQ obtained by the current method satisfies the minimum quantification limit of less than $0.05 \mathrm{mg} / \mathrm{kg}$ or less or $1 / 2$ of the residual tolerance standard, the pesticide residues analysis standard in the international standard Codex [28], and the Pesticide Residue Analysis Standard in the MFDA [21]. The analytical quantitation limit calculated in the current method was consistent with the minimum quantification limit of less than $0.05 \mathrm{mg} / \mathrm{kg}$ or less set by FAO codex Alimentarius or $1 / 2$ of the residual tolerance standard set by MFDS $[21,28]$. To evaluate the accuracy, precision, and repeatability of the tested method standard solution containing the limit of quantitation $(0.04 \mathrm{mg} / \mathrm{L})$ and 10 times the limit of quantification $(0.4 \mathrm{mg} / \mathrm{L})$ was added to the untreated samples. The average recovery rates were $88.8-106.3 \%$ and $83.1-88.4 \%$, and the RSD was generally good at a maximum of $8.8 \%$, indicating that the current method is sensitive and selective. Furthermore, the ranges of the recovery rate and analysis error satisfy the $70-120 \%$ of the single component analysis method of the MFDS, and the result satisfies the value within $10 \%$ of the RSD (Table S4).

$0.04(\mathrm{ng}) \times \frac{50(\mathrm{~mL})}{5(\mathrm{~g})} \times \frac{1(\mathrm{~mL})}{2(\mathrm{~mL})} \times \frac{1}{5(\mu \mathrm{L})}=0.04(\mathrm{mg} / \mathrm{kg})$

The improved analysis method was used to analyze kasugamycin residues (Fig. 8) in A. japonica and agricultural products. The kasugamycin analysis method developed in this study is expected to be applied to the

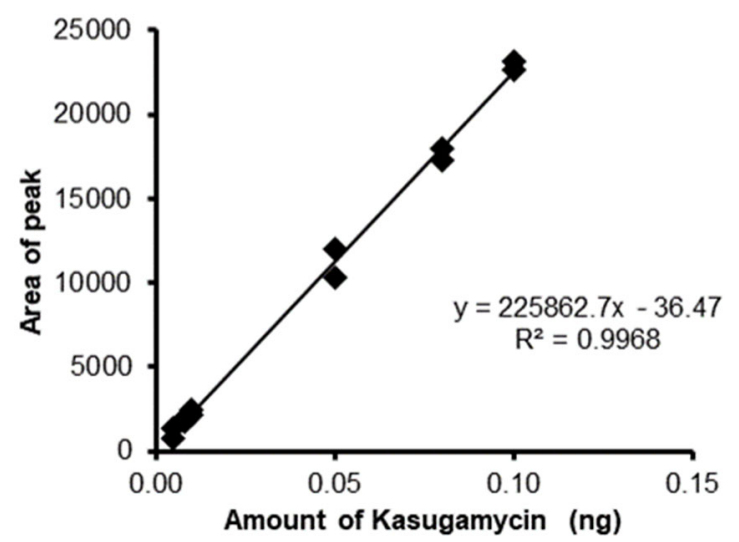

Fig 7. Calibration curve of kasugamycin matrixmatched standard by LC/MS/MS

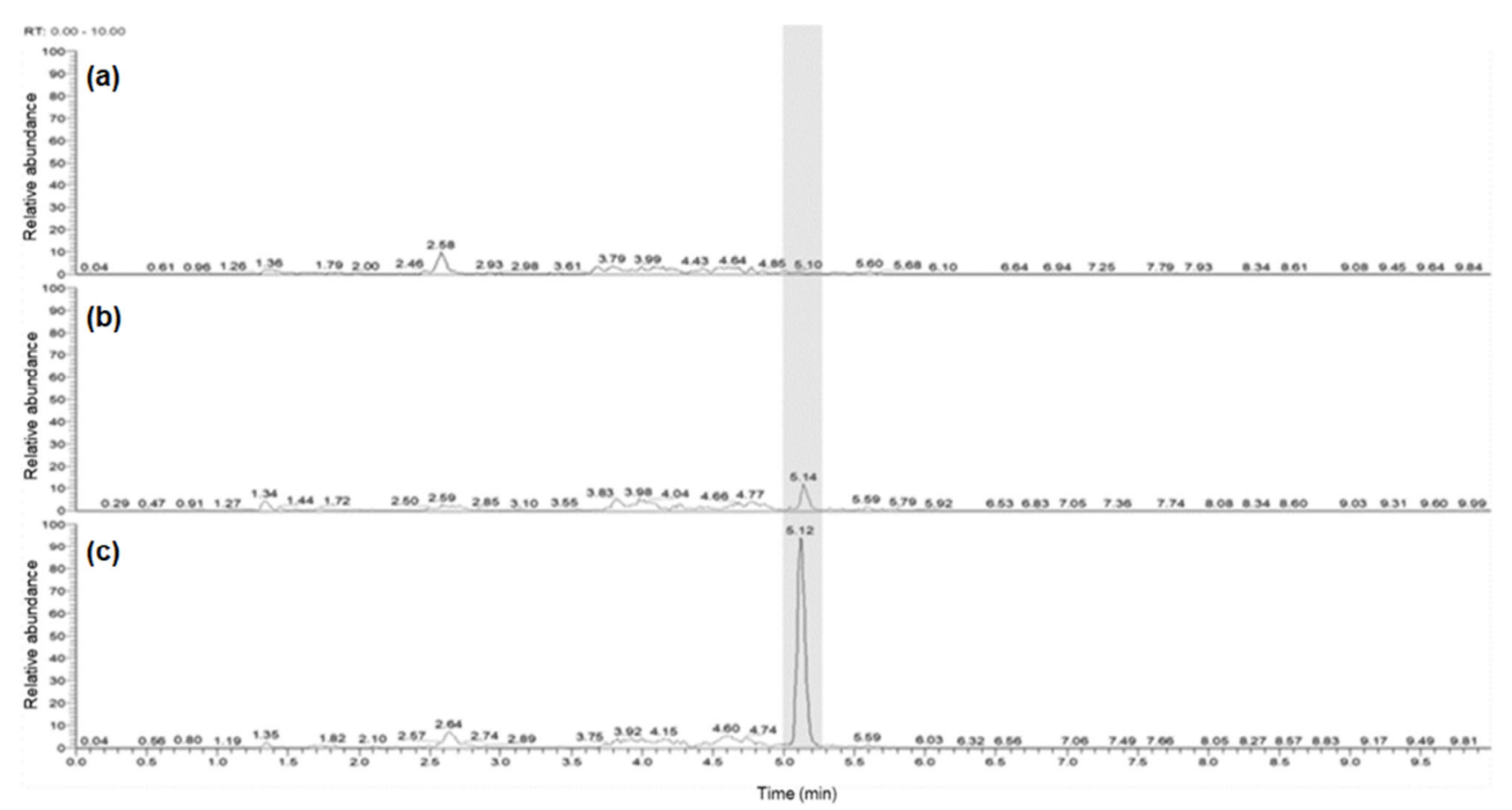

Fig 8. Chromatograms of kasugamycin residue analysis in Achyranthes japonica Nakai ((a): control, (b): 1 MLOQ (0.04 $\left.\left.\mathrm{mg} \mathrm{kg}^{-1}\right),(\mathrm{c}): 10 \operatorname{MLOQ}\left(0.4 \mathrm{mg} \mathrm{kg}^{-1}\right)\right)$ 
aminoglycoside-based agricultural fungicides and bactericides in various herbal medicines. Furthermore, it could also be applied to analyze the residues of other fungicides and bactericides in agricultural, livestock, and aquatic products, which are major food sources in the Republic of Korea and other countries. Therefore, this method may contribute to human safety.

\section{- CONCLUSION}

A suitable analytical method for the determination of the trace residue of kasugamycin in A. japonica was developed. The developed method involved using LC/MS/MS equipped with an amide column for polar substances with efficient extraction and purification processes optimized using a single SCX SPE cartridge, which is simple and time-efficient. The developed purification and cleanup method showed high efficiency in removing interferences with respective matrix effects while maintaining a high recovery rate of kasugamycin. The current results are consistent with the criteria ranges indicated in the guidelines of the Codex (CAC/GL 401993, 2003) and MFDS (2016). Furthermore, currently developed kasugamycin analysis could be applied to the aminoglycoside-based agricultural fungicides and bactericides and also the residues of other fungicides and bactericides. Therefore, this method may contribute to human safety.

\section{- ACKNOWLEDGMENTS}

This research was conducted with the Ministry of Food and Drug Safety funding in 2020 (19172 herbal medicines 196), and the authors are grateful for this.

\section{- AUTHOR CONTRIBUTIONS}

Jang Hyun is the team leader who proposed the work and designed the experiment. Jeong Yoon, Minwoo, and Hun Ju executed the experiments and analyzed the results. Jang Hyun and Abd Elaziz wrote the experiment. The first author (Jeong Yoon Choi) and the second author (Hun Ju Ham) have equal contributions. All authors read and approved the manuscript.

\section{- REFERENCES}

[1] Na, E.S., Kim, S.S., Hong, S.S., Kim, K.J., Lee, Y.J., Lee,
B.C., and Lee, K.S., 2020, Development of Multiresidue analytical method for 261 pesticides in herbal medicines using GC-MS/MS and LCMS/MS, Korean J. Environ. Agric., 39 (2), 142-169.

[2] Kang, M.Y., Lee, S.H., Lee, S.W., Cha, S.W., Song, J.L., and Lee, S.C., 2015, Effect of Achyranthis radix and Drynariae rhizoma extracts on antioxidant activity and antioxidant enzymes, Korean J. Plant Resour., 28 (5), 600-607.

[3] Rural Development Administration, 2021, Pesticide Safety Information System (PSIS), Rural Development Administration, Jeonju, Korea, http://psis.rda.go.kr/psis/agc/res/agchmRegistStus Lst.ps.

[4] Kim, S.H., and Park, Y.H., 2008, Antimicrobial resistance and food safety, Safe Food, 3 (1), 30-36.

[5] Park, J.S., Do, J.A., Lee, H. S., Park, S.M., Cho, S.M., Shin, H.S., Jang, D.E., Cho, M.S., Jung, Y.H., and Lee, K., 2019, Development of analytical method for detection of fungicide validamycin $\mathrm{A}$ residues in agricultural products using LC-MS/MS, J. Food Hyg. Saf., 34 (1), 22-29.

[6] Kim, T.J., Kim, K.S., Yoon, C.H., Joo, J.B., and Kim, C.H., 1997, Analysis of kasugamycin in pesticides formulations by reversed-phase ion pair liquid chromatography, Anal. Sci. Technol., 10 (5), 343349.

[7] Li, W., Dai, X., Pu, E., Bian, H., Chen, Z., Zhang, X., Guo, Z., Li, P., Li, H., Yong, Y., Wang, C., Zhang, Y., and Han, L., 2020, HLB-MCX-based solid-phase extraction combined with liquid chromatographytandem mass spectrometry for the simultaneous determination of four agricultural antibiotics (kasugamycin, validamycin $\mathrm{A}$, ningnanmycin, and polyoxin b) residues in plant-origin foods, J. Agric. Food Chem., 68 (47), 14025-14037.

[8] Zhang, H., Wang, C., Li, H., Nie, Y., Fang, L., and Chen, Z., 2017, Simultaneous determination of kasugamycin and validamycin-A residues in cereals by consecutive solid-phase extraction combined with liquid chromatography-tandem mass spectrometry, Food Addit. Contam., Part A, 35 (3), 487-497. 
[9] Isoherranen, N., and Soback, S., 1999, Chromatographic methods for analysis of aminoglycoside antibiotics, J. AOAC Int., 82 (5), 1017-1045.

[10] Lu, L., Zhao, S., Deng, L., Chen, Y., Liu, X., and Li., D., 2012, Residues and dynamics of kasugamycin in chilli and soil, Bull. Environ. Contam. Toxicol., 89 (3), 649-653.

[11] Alechaga, É., Moyano, E., and Galceran, M.T., 2015, Simultaneous analysis of kasugamycin and streptomycin in vegetables by liquid chromatography-tandem mass spectrometry, Anal. Methods, 7 (8), 3600-3607.

[12] Lee, H.S., Do, J.A., Park, J.S., Cho, S.M., Shin, H.S., Jang, D.E., Jung, Y.H., and Lee, K., 2019, Development of analytical method for kasugamycin in agricultural products using LC-MS/MS, J. Food Hyg. Saf., 34 (3), 235-241.

[13] Ministry of Food and Drug Safety Guidelines (MFDSG), 2016, Standard Procedure for Preparing Testing Methods for Food, etc. Test Guidelines, South Korea's Ministry of Food and Drug Safety.

[14] Turner, J.A., 2015, The Pesticide Manual, $17^{\text {th }}$ Ed., BPC Publications, Alton, Hampshire, UK.

[15] Zhou, W., Yang, S., and Wang, P.G., 2017, Matrix effects and application of matrix effect factor, Bioanalysis, 9 (23), 1839-1844.

[16] Ministry of Food and Drug Safety (MFDS), 2013, Pesticide Analytical Residues Manual in Food Code, 13-14.

[17] Chemicalize, 2021, Kasugamycin, https://chemicalize. com/\#/calculation, accessed on 23 August 2021.

[18] Arsand, J.B., Jank, L., Martins, M.T., Hoff, R.B., Barreto, F., Pizzolato, T.M., and Sirtori., C., 2016, Determination of aminoglycoside residues in milk and muscle based on a simple and fast extraction procedure followed by liquid chromatography coupled to tandem mass spectrometry and time of flight mass spectrometry, Talanta, 154, 38-45.

[19] Wang, C., Li, H., Wang, N., Li, H., Fang, L., Dong, Z., Du, H., Guan, H., Zhu, Q., Chen, Z., and Yang, G., 2017, Simultaneous analysis of kasugamycin and validamycin-1 $\mathrm{A}$ in fruits and vegetables using liquid chromatography-tandem mass spectrometry and consecutive solid-phase extraction, Anal. Methods, 9 (4), 634-642.

[20] New, L.S. and Chan, E.C.Y., 2008, Evaluation of BEH $\mathrm{C}_{18}$, BEH HILIC, and HSS T3 $\left(\mathrm{C}_{18}\right)$ column chemistries for the UPLC-MS-MS analysis of glutathione, glutathione disulfide, and ophthalmic acid in mouse liver and human plasma, $J$. Chromatogr. Sci., 46 (3), 209-214.

[21] Asakawa, D., Uemura, M., Sakiyama, T., and Yamano, T., 2017, Sensitivity enhancement of aminoglycosides in hydrophilic interaction liquid chromatography with tandem mass spectrometry by post-column addition of trace sodium acetate in methanol, Food Addit. Contam., Part A, 35 (6), 1116-1126.

[22] Ministry of Food and Drug Safety (MFDS), 2017, Guideline for Pesticide Residue Analysis Practice in the Korean Food Public Code, $4^{\text {th }}$ Ed, Osong, Republic of Korea.

[23] Kim, J.H., Moon, S.E., Kim, K.Y., Jung, Y.J., Lee, C.H., Ku, E.J., Yoon, M.H., and Lee, J.B., 2016, Simultaneous analysis for veterinary drug residues in honey by HPLC/MS/MS, J. Food Hyg. Saf., 31 (2), 94-98.

[24] Fontanals, N., Zohar, J., Borrull, F., Ronka, S., and Marcé, R.M., 2021, Development of a maleic acidbased material to selectively solid-phase extract basic compounds from environmental samples, $J$. Chromatogr. A, 1647, 462165.

[25] Fontanals, N., Marcé, R.M., and Borrull, F., 2019, Materials for solid-phase extraction of organic compounds, Separations, 6 (4), 56.

[26] Choi, J.Y., Lee, Y.J., Ham, H.J., Ishag, A.E.S.A., and Hur, J.H., 2021, A study on improvement of the analytical method of chlorantraniliprole residue in herbal medicine (Rehmannia glutinosa Libosch) using HPLC-UVD, Korean J. Pestic. Sci., 25 (3), 196-211.

[27] Bierman, S., and Campognone, M.V., 2009, Simplifying solid-phase extraction method development: exploring the use of software, LCGC, 27 (8), 24-26. 
[28] Joint FAO/WHO Codex Alimentarius Commission, 2003, Codex Alimentarius: Guidelines on Good Laboratory Practice in Pesticide Residue Analysis,
World Health Organization: Food and Agriculture Organization of the United Nations, Rome, CAC/GL 40-1993, 1-36. 